\title{
Event-by-Event Continuous Respiratory Motion Correction for Dynamic PET Imaging
}

\author{
Yunhan $\mathrm{Yu}^{1,2}$, Chung Chan ${ }^{1}$, Tianyu $\mathrm{Ma}^{2}$, Yaqiang $\mathrm{Liu}^{2}$, Jean-Dominique Gallezot ${ }^{1}$, Mika Naganawa ${ }^{1}$, Olivia J. Kelada ${ }^{3,4}$, \\ Mary Germino $^{5}$, Albert J. Sinusas ${ }^{1,6}$, Richard E. Carson ${ }^{1,5}$, and Chi Liu ${ }^{1,5}$ \\ ${ }^{I}$ Department of Diagnostic Radiology, Yale University, New Haven, Connecticut; ${ }^{2}$ Department of Engineering Physics, Key \\ Laboratory of Particle and Radiation Imaging (Tsinghua University), Ministry of Education, Beijing, China; ${ }^{3}$ Department of \\ Therapeutic Radiology, Yale University School of Medicine, New Haven, Connecticut; ${ }^{4}$ Department of Medical Physics in Radiation \\ Oncology, German Cancer Research Center, Heidelberg, Germany; ${ }^{5}$ Department of Biomedical Engineering, Yale University School \\ of Medicine, New Haven, Connecticut; and ${ }^{6}$ Department of Internal Medicine, Yale University School of Medicine, New Haven, \\ Connecticut
}

\begin{abstract}
Existing respiratory motion-correction methods are applied only to static PET imaging. We have previously developed an eventby-event respiratory motion-correction method with correlations between internal organ motion and external respiratory signals (INTEX). This method is uniquely appropriate for dynamic imaging because it corrects motion for each time point. In this study, we applied INTEX to human dynamic PET studies with various tracers and investigated the impact on kinetic parameter estimation. Methods: The use of 3 tracers-a myocardial perfusion tracer, ${ }^{82} \mathrm{Rb}$ $(n=7)$; a pancreatic $\beta$-cell tracer, ${ }^{18} \mathrm{~F}-\mathrm{FP}(+) \mathrm{DTBZ}(n=4)$; and a tumor hypoxia tracer, ${ }^{18} \mathrm{~F}$-fluoromisonidazole $\left({ }^{18} \mathrm{~F}-\mathrm{FMISO}\right)(n=1)$-was investigated in a study of 12 human subjects. Both rest and stress studies were performed for ${ }^{82} \mathrm{Rb}$. The Anzai belt system was used to record respiratory motion. Three-dimensional internal organ motion in high temporal resolution was calculated by INTEX to guide event-by-event respiratory motion correction of target organs in each dynamic frame. Time-activity curves of regions of interest drawn based on endexpiration PET images were obtained. For ${ }^{82} \mathrm{Rb}$ studies, $K_{1}$ was obtained with a 1-tissue model using a left-ventricle input function. Rest-stress myocardial blood flow (MBF) and coronary flow reserve (CFR) were determined. For ${ }^{18} \mathrm{~F}-\mathrm{FP}(+)$ DTBZ studies, the total volume of distribution was estimated with arterial input functions using the multilinear analysis 1 method. For the ${ }^{18} \mathrm{~F}$-FMISO study, the net uptake rate $K_{\mathrm{i}}$ was obtained with a 2-tissue irreversible model using a left-ventricle input function. All parameters were compared with the values derived without motion correction. Results: With INTEX, $K_{1}$ and MBF increased by $10 \% \pm 12 \%$ and $15 \% \pm 19 \%$, respectively, for ${ }^{82} \mathrm{Rb}$ stress studies. CFR increased by $19 \% \pm 21 \%$. For studies with motion amplitudes greater than $8 \mathrm{~mm}(n=3), K_{1}, \mathrm{MBF}$, and CFR increased by $20 \% \pm 12 \%, 30 \% \pm 20 \%$, and $34 \% \pm 23 \%$, respectively. For ${ }^{82} \mathrm{Rb}$ rest studies, INTEX had minimal effect on parameter estimation. The total volume of distribution of ${ }^{18} \mathrm{~F}$ $\mathrm{FP}(+)$ DTBZ and $K_{\mathrm{i}}$ of ${ }^{18} \mathrm{~F}$-FMISO increased by $17 \% \pm 6 \%$ and $20 \%$, respectively. Conclusion: Respiratory motion can have a substantial impact on dynamic PET in the thorax and abdomen. The INTEX method using continuous external motion data substantially changed parameters in kinetic modeling. More accurate estimation is expected with INTEX.
\end{abstract}

Received Oct. 9, 2015; revision accepted Feb. 1, 2016.

For correspondence or reprints contact: Chi Liu, Department of Diagnostic Radiology, Yale University, P.O. Box 208048, New Haven, CT 06520-8048. E-mail: chi.liu@yale.edu

Published online Feb. 23, 2016.

COPYRIGHT (c) 2016 by the Society of Nuclear Medicine and Molecular Imaging, Inc.
Key Words: respiratory motion correction; dynamic PET imaging; INTEX

J Nucl Med 2016; 57:1084-1090

DOI: 10.2967/jnumed.115.167676

\section{$\mathbf{R}$} espiratory motion is a major challenge in PET imaging, leading to image quality degradation including blurring and contrast reduction (1), as well as quantitative errors in regional radioactivity concentration measurements (2), which can lead to inaccuracies in tumor delineation and errors in clinical diagnoses (3).

In dynamic PET imaging, which aims to extract physiologically relevant information from multiple time frames instead of 1 static image, respiratory motion could result in quantitative errors in each individual time frame. Moreover, irregular breathing patterns and amplitude variations during the dynamic scan may lead to framedependent motion blurring, further confounding parameter estimation from kinetic modeling.

In cardiac PET, the estimation of coronary flow reserve (CFR) requires 2 dynamic PET scans, one at rest and the other during pharmacologic stress. When vasodilators are administered, the patients' breathing pattern can change substantially. As shown in Figure 1, the amplitude of respiratory motion during the stress scan is substantially larger than that during the rest scan for the same subject. Different amounts of motion blurring occur in association with rest and stress scans and can lead to errors in myocardial blood flow (MBF) quantification and confound the assessment of CFR. Therefore, respiratory motion correction (MC) can be critical for dynamic PET imaging.

Numerous methods have been proposed to compensate for respiratory motion in static PET imaging (4). With respiratory motion tracked by external devices or motion information extracted from data-driven methods, MC is typically conducted by gating strategies (5), image-based registration (6), or motion-compensated image reconstruction (7).

Respiratory gating is the most common correction approach in static PET imaging. However, with gated dynamic imaging, each reconstructed image has higher image noise as only a small fraction of counts is allocated to each frame (8). When all of the detected events are used, registration of dynamic gated images into motion-compensated dynamic images is challenging especially 


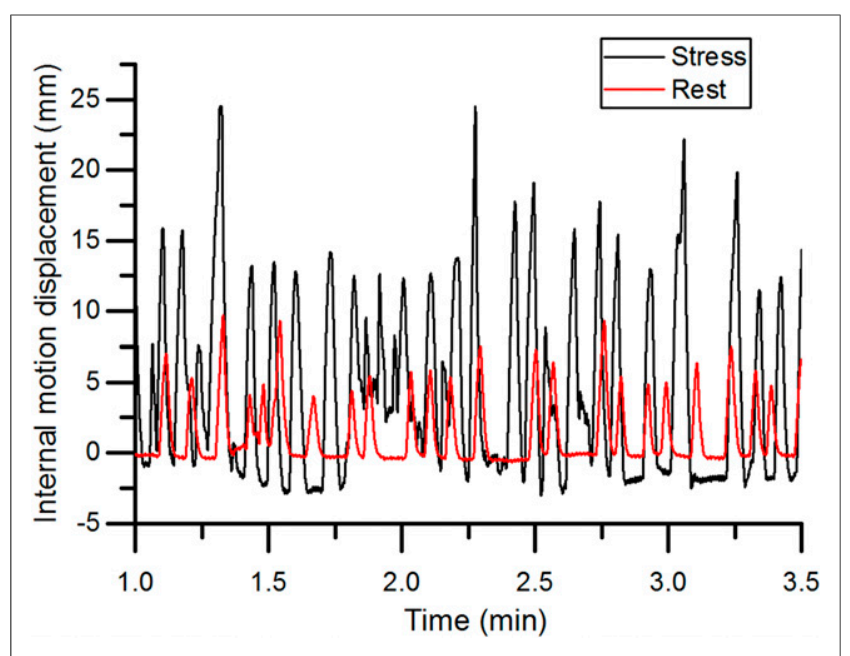

FIGURE 1. Internal motion displacement of myocardium caused by respiration for 1 subject during both rest and regadenosine-induced stress imaging with ${ }^{82} \mathrm{Rb}$.

for images of early frames. Those early images between 0 and 3 min may demonstrate rapid changes in the radiotracer distribution and fewer visible structures. Reliable motion vectors typically can be estimated only from delayed gated images. The same challenge holds for motion-compensated image reconstruction.

We have previously developed an MC method using internalexternal (INTEX) motion correlation $(9,10)$ that is integrated with a motion-compensation ordered-subsets expectation maximization list-mode algorithm for resolution-recovery reconstruction (MOLAR) $(11,12)$. This method performs continuous respiratory MC on an event-by-event basis, thus is capable of correcting respiratory motion in dynamic imaging. We have applied this approach to static PET images in previous studies $(9,10)$. In this study, we applied this INTEX method to 12 dynamic human PET studies with 3 different tracers and investigated the effects on quantitation of kinetic parameters.

\section{MATERIALS AND METHODS}

\section{Overview of INTEX MC}

A brief overview of INTEX is given here, although the details of the methodology have been previously published $(9,10)$. In this method, we first derived linear correlations between the mean displacements of the 1-dimensional external respiratory trace acquired by the Anzai system (Anzai Medical) for each gate and the centroid of an internal target organ (e.g., heart, tumor) derived from gated images in 3 dimensions. On the basis of the correlations, a continuous internal motion file was then generated by converting the entire external trace into a 3-dimensional (3D) transformation matrix with high temporal resolution $(\geq 40 \mathrm{~Hz})$ to guide event-by-event respiratory MC. This is accomplished by rigidly relocating the line of response of each event in MOLAR reconstruction. The INTEX approach corrects respiratory motion using all detected events without increasing image noise. The event-by-event MC can effectively eliminate intragate motion caused by both intercycle and intracycle variations and is uniquely capable of correcting framedependent motion for dynamic PET dataset.

\section{Experiments and Data Acquisition}

To comprehensively evaluate the proposed INTEX approach for respiratory $\mathrm{MC}$ of dynamic PET images, 12 human subjects were imaged using 3 different radiotracers with different clinical tasks. All studies were approved by the Yale University Human Investigation Committee and Radiation Safety Committee.

Seven healthy subjects were included for cardiac dynamic PET imaging with ${ }^{82} \mathrm{Rb}$, with the goal of quantification of MBF and determination of CFR (13). Both rest and regadenoson-induced stress scans were acquired. Vasodilation was induced with a $0.4-\mathrm{mg}$ dose of regadenoson, injected over $30 \mathrm{~s}$, approximately $1 \mathrm{~min}$ before the ${ }^{82} \mathrm{Rb}$ infusion was initiated using a CardioGen-82 infusion system (Bracco Diagnostics) with an infusion rate of $50 \mathrm{~mL} / \mathrm{min}$. The average injected ${ }^{82} \mathrm{Rb}$ dose was $670 \pm 67 \mathrm{MBq}$. For attenuation correction, a noncontrast low-dose CT scan was obtained before the rest PET scan, and another noncontrast CT scan was obtained immediately after the stress PET scan. List-mode PET data acquired over the first $4 \mathrm{~min}$ after injection were reconstructed and analyzed for kinetic modeling.

Abdominal PET imaging studies were acquired in 4 subjects injected with ${ }^{18} \mathrm{~F}$-labeled 9-fluoropropyl-(+)- ${ }^{11} \mathrm{C}$-dihydrotetrabenazine

TABLE 1

Summary of Subject Demographics and Associated Internal Organ Motion Amplitudes

\begin{tabular}{lcccccc}
\hline Subject no. & Tracer & Sex & Age $(\mathrm{y})$ & Weight $(\mathrm{kg})$ & $\begin{array}{c}\text { Motion amplitude (mm) } \\
\left.\text { (stress-rest for }{ }^{82} \mathrm{Rb}\right)\end{array}$ & Health status \\
\hline 1 & ${ }^{82} \mathrm{Rb}$ & $\mathrm{F}$ & 32 & 63 & $13.3 / 5.8$ & Healthy \\
2 & ${ }^{82} \mathrm{Rb}$ & $\mathrm{M}$ & 24 & 95 & $12.4 / 6.9$ & Healthy \\
3 & ${ }^{82} \mathrm{Rb}$ & $\mathrm{F}$ & 32 & 81 & $11.3 / 7.8$ & Healthy \\
\hline 4 & ${ }^{82} \mathrm{Rb}$ & $\mathrm{F}$ & 26 & 53 & $7.0 / 4.1$ & Healthy \\
\hline 5 & ${ }^{82} \mathrm{Rb}$ & $\mathrm{M}$ & 34 & 71 & $6.8 / 7.0$ & Healthy \\
\hline 6 & ${ }^{82} \mathrm{Rb}$ & $\mathrm{M}$ & 31 & 92 & $6.2 / 5.5$ & Healthy \\
\hline 7 & ${ }^{82} \mathrm{Rb}$ & $\mathrm{M}$ & 37 & 65 & $4.2 / 4.6$ & Healthy \\
\hline 8 & ${ }^{18} \mathrm{~F}-\mathrm{FP}(+) \mathrm{DTBZ}$ & $\mathrm{M}$ & 39 & 62 & 12.6 & Healthy \\
\hline 10 & ${ }^{18} \mathrm{~F}-\mathrm{FP}(+) \mathrm{DTBZ}$ & $\mathrm{M}$ & 31 & 87 & 10.3 & Healthy \\
11 & ${ }^{18} \mathrm{~F}-\mathrm{FP}(+) \mathrm{DTBZ}$ & $\mathrm{M}$ & 27 & 76 & 9.7 & Diabetic patient \\
\hline 12 & ${ }^{18} \mathrm{~F}-\mathrm{FP}(+) \mathrm{DTBZ}$ & $\mathrm{M}$ & 24 & 74 & 7.4 & Healthy \\
\hline
\end{tabular}


$\left({ }^{18} \mathrm{~F}-\mathrm{FP}(+) \mathrm{DTBZ}\right)$, a radiotracer that binds to the vesicular monoamine transporter- 2 in $\beta$-cells in the pancreas (14). These subjects were imaged twice over a 240-min period after injection. The first PET scan was acquired for a 2-h period. After a 30-min break, during which the subjects rested, a second 90-min scan was acquired. The average injected dose was $224 \pm 60 \mathrm{MBq}$. To provide an input function, serial arterial blood samples were collected for determination of metabolites by high-performance liquid chromatography. CT data were acquired before each PET scan for attenuation correction.

One patient with non-small cell lung cancer underwent PET imaging of the chest after injection of $180 \mathrm{MBq}$ of ${ }^{18} \mathrm{~F}$-fluoromisonidazole $\left({ }^{18} \mathrm{~F}\right.$-FMISO), a hypoxia-targeted radiotracer (15). Two PET images were acquired over $180 \mathrm{~min}$ after injection. The first scan was acquired over $120 \mathrm{~min}$ and was followed by a second $30-\mathrm{min}$ scan after a 30-min break. CT data were acquired before each PET scan.

\section{Imaging Protocol}

All PET data were acquired in list mode on a 3D scanner Biograph mCT; Siemens) (16). For each subject, the first PET scan began shortly before the radiotracer injection to capture the early phase of the tracer kinetics. External respiratory motion was tracked by the Anzai belt system, which was attached to the patients' lower abdomen. The respiratory trace was first recorded at $40 \mathrm{~Hz}$ for subjects (subjects 4 and 7-12) and then improved to $50 \mathrm{~Hz}$ (subjects 1-3 and 5) after a software upgrade.

\section{INTEX MC for Dynamic PET}

$3 D$ Internal-External Correlation. The relative amplitude of the Anzai respiratory waveform was reset at the beginning of each PET scan. Therefore, internal-to-external correlations were built separately for each individual PET acquisition in each subject. Taking the ${ }^{18} \mathrm{~F}-\mathrm{FP}(+) \mathrm{DTBZ}$ PET studies as an example, 2 different correlations were generated. One correlation was generated for the first 2 -h scan and a second for the follow-up 90-min scan. If the Anzai system was restarted during a scan for any reason, for example, because of the need to readjust the belt to accommodate for drift in the breathing pattern, the correlation was also rebuilt from that time point.

Gated ${ }^{82} \mathrm{Rb}$ PET images were reconstructed from data acquired between 2 and $6 \mathrm{~min}$ after injection. For generation of gated ${ }^{18} \mathrm{~F}-\mathrm{FP}(+) \mathrm{DTBZ}$ and ${ }^{18} \mathrm{~F}$-FMISO images, the first $30 \mathrm{~min}$ of data of each PET scan were used. All gated images were reconstructed using the previously published point-spread function and time-of-flight ordered-subsets expectation maximization method (17) with 2 iterations and 30 subsets using $2 \times 2 \times 2 \mathrm{~mm}$ voxels. For every gated PET image of the same subject, the matched CT image was used for attenuation correction, as explained in the next section.

Using a previously reported level-set method (18) to segment target organs (subjects $1-7$, myocardium; subjects $8-11$, pancreas; subject 12 , tumor in lung) from gated images, we established linear correlations between internal organ motion and measured external motion as described above. The correlations extracted from each scan were then applied to each list-mode file. A pilot study (described in the supplemental data [available at http://jnm.snmjournals.org]) indicated that the difference in the impact of correlation changes for INTEX within 1 scan was small, though additional investigations may be warranted.

The internal motion amplitudes of the target organ were also determined by calculating the difference in the center of mass of the organs obtained from the end-inspiration and end-expiration images.

Matched Attenuation Correction. All CT attenuation images were acquired during an end-expiration breath hold. For MC, the reference PET frame was also chosen at the end-expiration phase to match with
Without motion correction With INTEX motion correction
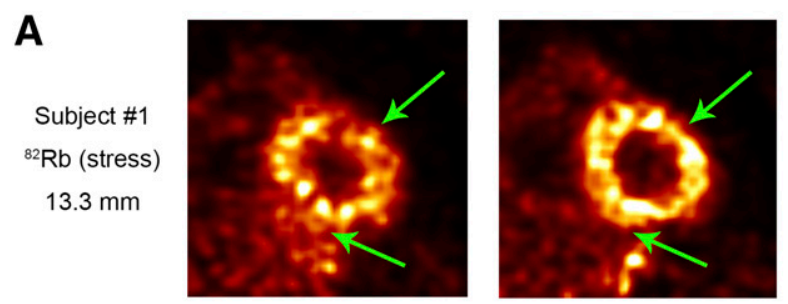

B
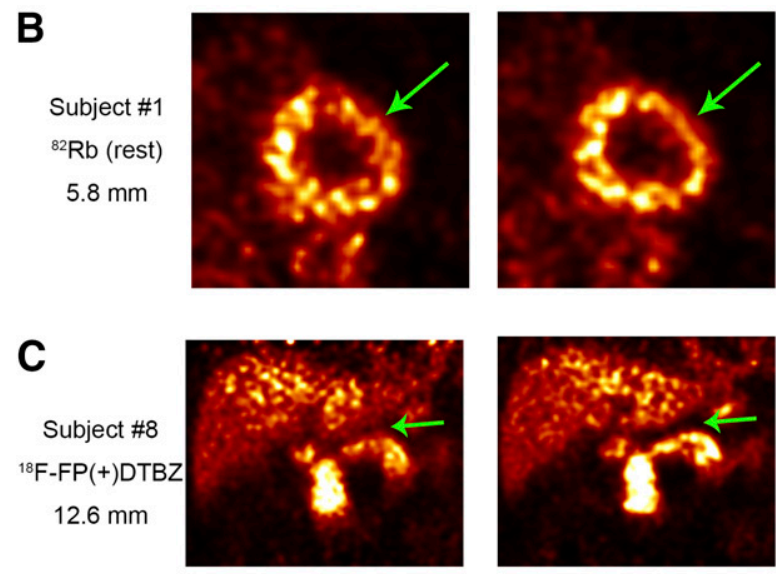

D
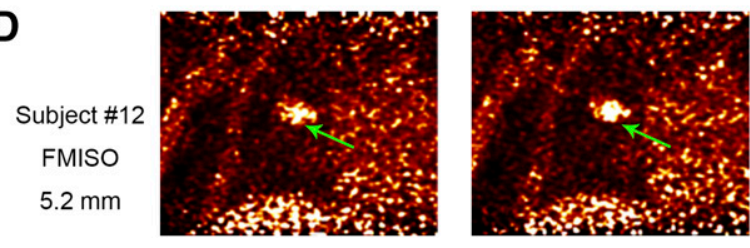

FIGURE 2. Images reconstructed without and with INTEX MC. (A) Images in sagittal view from 2- to 6 -min frames for ${ }^{82} \mathrm{Rb}$ subject 1 stress study. (B) Images in sagittal view from 2- to 6 -min frames for ${ }^{82} \mathrm{Rb}$ subject 1 rest study. (C) Images in coronal view from 150- to 155-min frames for subject $8{ }^{18} \mathrm{~F}-\mathrm{FP}(+) \mathrm{DTBZ}$ study. (D) Images in coronal view from 150 - to 155 -min frames for subject $12{ }^{18} \mathrm{~F}$-FMISO study. Images in each row are displayed on same scale.

the attenuation-correction scans. Specifically, because each line of response is repositioned to the location at end-expiration during list-mode reconstruction, the matched attenuation correction will be achieved for all events. Manual rigid registration was performed between end-expiration gated PET and attenuation-correction CT images if residual mismatch was observed.

Dynamic PET Data Generation. Thirty-two dynamic ${ }^{82} \mathrm{Rb}$ frames $(3 \mathrm{~s} \times 20,10 \mathrm{~s} \times 6,20 \mathrm{~s} \times 6), 49^{18} \mathrm{~F}-\mathrm{FP}(+)$ DTBZ frames $(30 \mathrm{~s} \times 6$, $1 \mathrm{~min} \times 3,2 \mathrm{~min} \times 2,5 \mathrm{~min} \times 38)$, and 39 FMISO frames $(30 \mathrm{~s} \times 6$, $1 \mathrm{~min} \times 3,2 \min \times 2,5 \min \times 28$ ) were reconstructed for each subject.

All dynamic images with and without INTEX MC were reconstructed by MOLAR with 2 iterations and 30 subsets using $2 \times 2 \times$ $2 \mathrm{~mm}$ voxels. Corrections for attenuation, scatter, random, and normalization were included in the reconstruction.

Three-dimensional regions of interest (ROIs) were defined from end-expiration PET images on the basis of the level-set segmentation. ROIs were also eroded slice by slice manually to the central part of target organ to minimize partial-volume effects. Time-activity curves of the target organ and left ventricle were then obtained. 


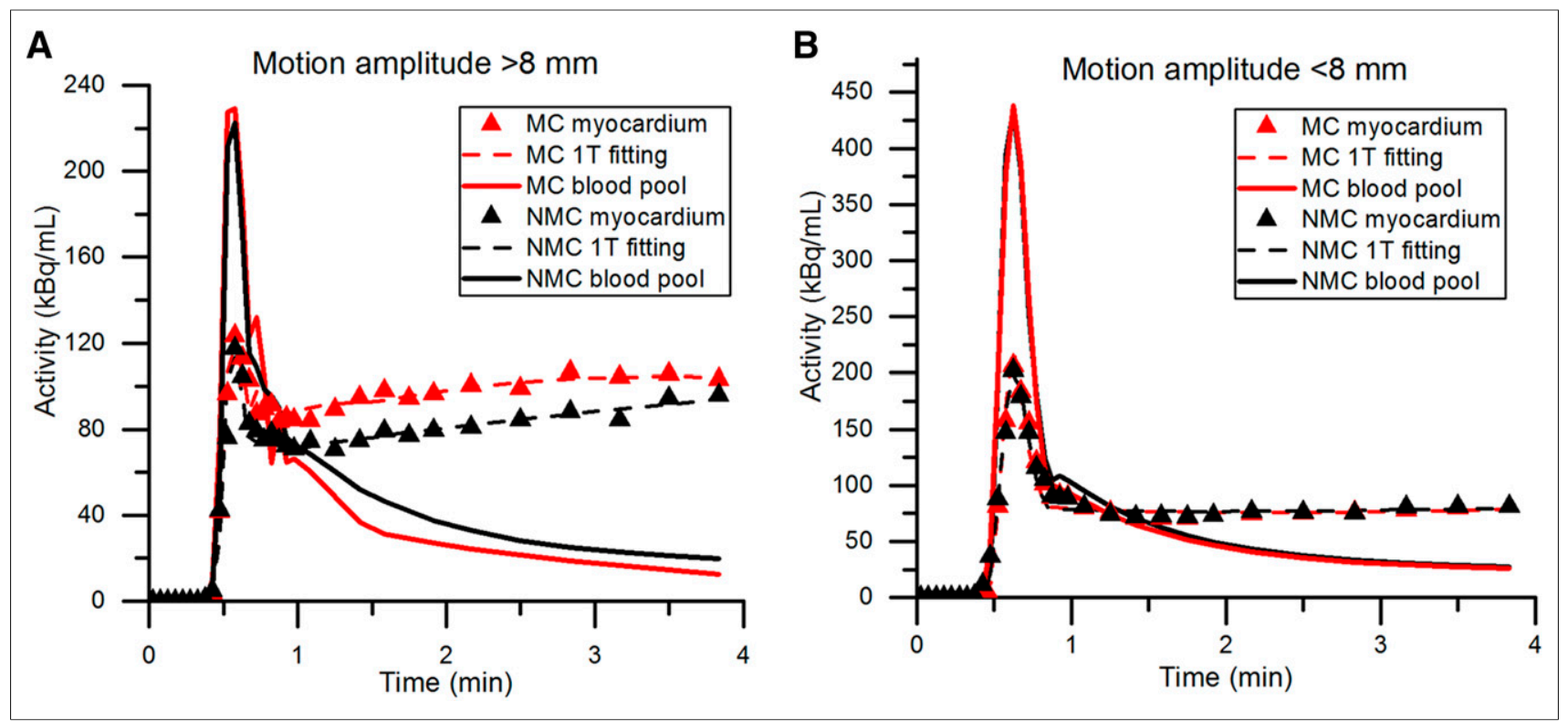

FIGURE 3. Time-activity curves of myocardium and blood pool of left ventricle for both MC and NMC analyses of subject 1 stress studies (motion amplitude, $13.3 \mathrm{~mm}$ ) (A) and subject 7 stress studies (motion amplitude, $4.2 \mathrm{~mm}$ ) (B). Data with $\mathrm{MC}$ are displayed in red and data without MC are in black. Triangles and solid line represent activity in myocardium and blood pool, respectively. Dashed line shows myocardial 1-tissue (1T) fitting result.

For ${ }^{82} \mathrm{Rb}$ studies, $K_{1}$ values were obtained with a 1-tissue model using a left-ventricle image-derived input function fitted to a 3-parameter model. Rest-stress global MBF and CFR were calculated on the basis of a previously published ${ }^{82} \mathrm{Rb}$ extraction function equation (13).

$$
\begin{gathered}
K_{1}=\left(1-0.77 \times e^{-0.63 / \mathrm{MBF}}\right) \times \mathrm{MBF} . \\
\mathrm{CFR}=\frac{\mathrm{MBF}_{\text {stress }}}{\mathrm{MBF}_{\text {rest }}} .
\end{gathered}
$$

Eq. 2

The total volume of distribution $\left(V_{\mathrm{T}}\right)$ of the pancreas was estimated with the arterial input function by the multilinear analysis 1 method for ${ }^{18} \mathrm{~F}-\mathrm{FP}(+) \mathrm{DTBZ}$ data (19).

A 2-tissue irreversible model was used for ${ }^{18} \mathrm{~F}$-FMISO with an input function derived from a left-ventricle ROI analysis to estimate the net uptake rate $K_{\mathrm{i}}$ from $K_{1} k_{3} /\left(k_{2}+k_{3}\right)(20)$.

Statistical Analysis. Percentage differences of the estimated parameters $\left(K_{1}, V_{\mathrm{T}}\right.$, and $K_{\mathrm{i}}$ for the 3 radiotracers) between data with no MC (NMC) and with $\mathrm{MC}$ were calculated as:

$$
\text { Percentage_difference }=\left(\frac{\mathrm{P}_{\mathrm{MC}}}{\mathrm{P}_{\mathrm{NMC}}}-1\right) \times 100 \% . \quad \text { Eq. } 3
$$

$P=K_{1}$ for subjects $1-7, P=V_{\mathrm{T}}$ for subjects $8-11$, and $P=K_{\mathrm{i}}$ for subject 12.

The $K_{1}, V_{\mathrm{T}}$, and $K_{\mathrm{i}}$ of all 12 subjects of both NMC and MC analyses were compared using nonparametric paired sign test, an alternative to the paired $t$ test appropriate for non-gaussian data.

\section{RESULTS}

Table 1 shows the demographic information from the subjects in this study. In addition, average respiratory motion amplitudes for the heart, pancreas, and lung tumor (Table 1) were $8.7 \pm$ $3.3 \mathrm{~mm}$ (stress)/6.0 $\pm 1.2 \mathrm{~mm}$ (rest), $9.9 \pm 2.1 \mathrm{~mm}$, and
$5.2 \mathrm{~mm}$, respectively. If multiple scans were obtained for the same subject, the average motion amplitudes across all scans are shown in the table.

Figure 2 shows representative static images of ${ }^{82} \mathrm{Rb}(2-6 \mathrm{~min})$, ${ }^{18} \mathrm{~F}-\mathrm{FP}(+)$ DTBZ (150-155 min), and ${ }^{18} \mathrm{~F}-\mathrm{FMISO}$ (150-155 min) without and with INTEX MC. MC visually reduced the respiratory blurring and radiotracer cross-talk in the areas indicated by the arrows. For the ${ }^{82} \mathrm{Rb}$ study, $\mathrm{MC}$ resulted in a greater reduction in blurring in the stress scan than in the rest scan.

Figure 3 shows sample time-activity curves of myocardium and left-ventricle blood pool for both MC and NMC analyses in 2 stress ${ }^{82} \mathrm{Rb}$ studies. For a subject (subject 1 in Table 1) with a motion amplitude of $13.3 \mathrm{~mm}$, INTEX changed the shape and

\section{TABLE 2}

$K_{1}$ Values for Stress and Rest Studies for Cardiac Subjects

\begin{tabular}{|c|c|c|c|c|c|c|c|c|}
\hline \multirow{3}{*}{$\begin{array}{c}\text { Subject } \\
\text { no. }\end{array}$} & \multirow{2}{*}{\multicolumn{2}{|c|}{$\begin{array}{l}\text { Motion } \\
\text { amplitude } \\
(\mathrm{mm})\end{array}$}} & \multicolumn{4}{|c|}{$\begin{array}{c}K_{1} \\
(\mathrm{~mL} / \mathrm{min} / \mathrm{g})\end{array}$} & \multirow{2}{*}{\multicolumn{2}{|c|}{$\begin{array}{l}\text { Percentage } \\
\text { difference }\end{array}$}} \\
\hline & & & \multicolumn{2}{|c|}{ NMC } & \multicolumn{2}{|c|}{ MC } & & \\
\hline & Stress & Rest & Stress & Rest & Stress & Rest & Stress & Rest \\
\hline 1 & 13.3 & 5.8 & 1.17 & 0.73 & 1.61 & 0.71 & $38 \%$ & $-4 \%$ \\
\hline 2 & 12.4 & 6.9 & 1.68 & 0.56 & 1.92 & 0.57 & $14 \%$ & $0 \%$ \\
\hline 3 & 11.3 & 7.8 & 1.40 & 0.80 & 1.53 & 0.77 & $10 \%$ & $-4 \%$ \\
\hline 4 & 7.0 & 4.1 & 1.33 & 0.75 & 1.37 & 0.76 & $3 \%$ & $2 \%$ \\
\hline 5 & 6.8 & 7.0 & 1.08 & 0.62 & 1.14 & 0.59 & $5 \%$ & $-4 \%$ \\
\hline 6 & 6.2 & 5.5 & 0.94 & 0.57 & 0.96 & 0.54 & $2 \%$ & $-6 \%$ \\
\hline 7 & 4.2 & 4.6 & 1.24 & 0.50 & 1.22 & 0.51 & $-1 \%$ & $1 \%$ \\
\hline Average & 8.7 & 6.0 & 1.26 & 0.65 & 1.39 & 0.63 & $10 \%$ & $-2 \%$ \\
\hline
\end{tabular}
Without and With MC As Well As Percentage Difference of $K_{1}$ Between $\mathrm{MC}$ and $\mathrm{NMC}$ 
TABLE 3

MBF of Both Stress and Rest Studies and CRF for 7 Cardiac Subjects Without and With MC As Well As Percentage Difference of MBF and CFR Between MC and NMC

\begin{tabular}{|c|c|c|c|c|c|c|c|c|c|}
\hline \multirow[b]{3}{*}{ Subject no. } & \multicolumn{4}{|c|}{$\mathrm{MBF}(\mathrm{mL} / \mathrm{min} / \mathrm{g})$} & \multirow{2}{*}{\multicolumn{2}{|c|}{$\begin{array}{c}\text { Percentage } \\
\text { difference of } \\
\text { MBF }\end{array}$}} & \multirow{2}{*}{\multicolumn{2}{|c|}{ CFR }} & \multirow{3}{*}{$\begin{array}{c}\text { Percentage difference } \\
\text { of CFR }\end{array}$} \\
\hline & \multicolumn{2}{|c|}{ NMC } & \multicolumn{2}{|c|}{ MC } & & & & & \\
\hline & Stress & Rest & Stress & Rest & Stress & Rest & NMC & $\mathrm{MC}$ & \\
\hline 1 & 3.17 & 1.46 & 5.02 & 1.39 & $58 \%$ & $-5 \%$ & 2.17 & 3.61 & $66 \%$ \\
\hline 2 & 5.31 & 0.91 & 6.34 & 0.94 & $19 \%$ & $3 \%$ & 5.84 & 6.74 & $16 \%$ \\
\hline 3 & 4.13 & 1.71 & 4.68 & 1.60 & $13 \%$ & $-6 \%$ & 2.42 & 2.93 & $21 \%$ \\
\hline 4 & 3.84 & 1.53 & 4.00 & 1.56 & $4 \%$ & $2 \%$ & 2.51 & 2.56 & $2 \%$ \\
\hline 5 & 2.81 & 1.09 & 3.05 & 1.00 & $9 \%$ & $-8 \%$ & 2.58 & 3.05 & $18 \%$ \\
\hline 6 & 2.25 & 0.94 & 2.32 & 0.86 & $3 \%$ & $-9 \%$ & 2.39 & 2.70 & $13 \%$ \\
\hline 7 & 3.46 & 0.77 & 3.38 & 0.80 & $-2 \%$ & $4 \%$ & 4.49 & 4.23 & $-6 \%$ \\
\hline Average & 3.57 & 1.20 & 4.11 & 1.16 & $15 \%$ & $-3 \%$ & 3.20 & 3.69 & $19 \%$ \\
\hline
\end{tabular}

values of both tissue and blood-pool time-activity curves (Fig. 3A), specifically increasing the later tissue values and decreasing the late blood-pool data. For another subject (subject 7 in Table 1) with a small motion amplitude $(4.2 \mathrm{~mm})$, the time-activity curve of NMC and MC nearly overlapped.

As shown in Table 2, overall, $K_{1}$ increased by $10 \% \pm 12 \%$ for stress studies with INTEX. For the subjects (subjects 1-3) with a motion amplitude greater than $8 \mathrm{~mm}, K_{1}$ increased by $20 \% \pm$ $12 \%$. For rest studies with smaller motion amplitudes, INTEX MC had minimal effect on $K_{1}$ estimation.

Table 3 summarizes the MBF and CFR values (Eqs. 1 and 2) and the changes in these values after MC was applied. MBF increased by $15 \% \pm 19 \%$ for stress studies with INTEX. For the studies with motion amplitudes greater than $8 \mathrm{~mm}$, MBF increased by $30 \% \pm 20 \%$.

CFR increased by $19 \% \pm 21 \%$ after MC on average. For subjects 4-7 with motion amplitudes less than $8 \mathrm{~mm}$ in both stress and rest studies, the increase on CFR was only $7 \% \pm 9 \%$ with $\mathrm{MC}$, whereas for subjects with motion amplitudes greater than $8 \mathrm{~mm}$, the increase was $34 \% \pm 23 \%$ on average, with a maximum change of $66 \%$.

Figure 4 shows the time-activity curves for ${ }^{18} \mathrm{~F}-\mathrm{FP}(+) \mathrm{DTBZ}$ subject 8 . MC resulted in a higher uptake in the pancreatic time-activity curve. As shown in Table 4, with INTEX MC, $V_{\mathrm{T}}$ for ${ }^{18} \mathrm{~F}-\mathrm{FP}(+) \mathrm{DTBZ}$ increased by $17 \% \pm 6 \%$. The standard errors of $V_{\mathrm{T}}$, as calculated from the theoretic parameter covariance matrix, were reduced by $28 \% \pm 33 \%$ on average.

Figure 5 shows time-activity curves of the lung tumor in the ${ }^{18}$ F-FMISO study. Internal motion amplitude averaged for every minute over the entire scan duration is also displayed. In the second scan, the average motion amplitude changed from 3.9 (0-120 $\mathrm{min})$ to $6.5 \mathrm{~mm}(150-180 \mathrm{~min})$, leading to larger increase of time-activity curve values with MC. With INTEX correction, $K_{\mathrm{i}}$ for ${ }^{18} \mathrm{~F}$-FMISO increased by $20 \%$ and the SE of $K_{\mathrm{i}}$ was reduced by $10 \%$.

Figure 6 plots the percentage difference of $K_{1}$ for ${ }^{82} \mathrm{Rb}, V_{\mathrm{T}}$ for ${ }^{18} \mathrm{~F}-\mathrm{FP}(+) \mathrm{DTBZ}$, and $K_{\mathrm{i}}$ for ${ }^{18} \mathrm{~F}-\mathrm{FMISO}$ in each study between the NMC and MC analysis as a function of internal motion amplitudes. A clear trend can be observed that larger respiratory motion amplitude leads to larger differences in parameter estimates between MC and NMC analyses. The $P$ value of nonparametric alternative to the paired sample $t$ test between the NMC and MC groups of kinetic parameters $\left(V_{\mathrm{T}}, K_{\mathrm{i}}, K_{1}\right)$ was 0.0076 , indicating that MC produced a significant increase in kinetic parameter estimates, consistent with less motion-induced blurring.

\section{DISCUSSION}

Respiratory motion is an important source of error in PET images. The INTEX method is capable of correcting respiratory motion for dynamic PET, whereas existing MC methods typically are applied

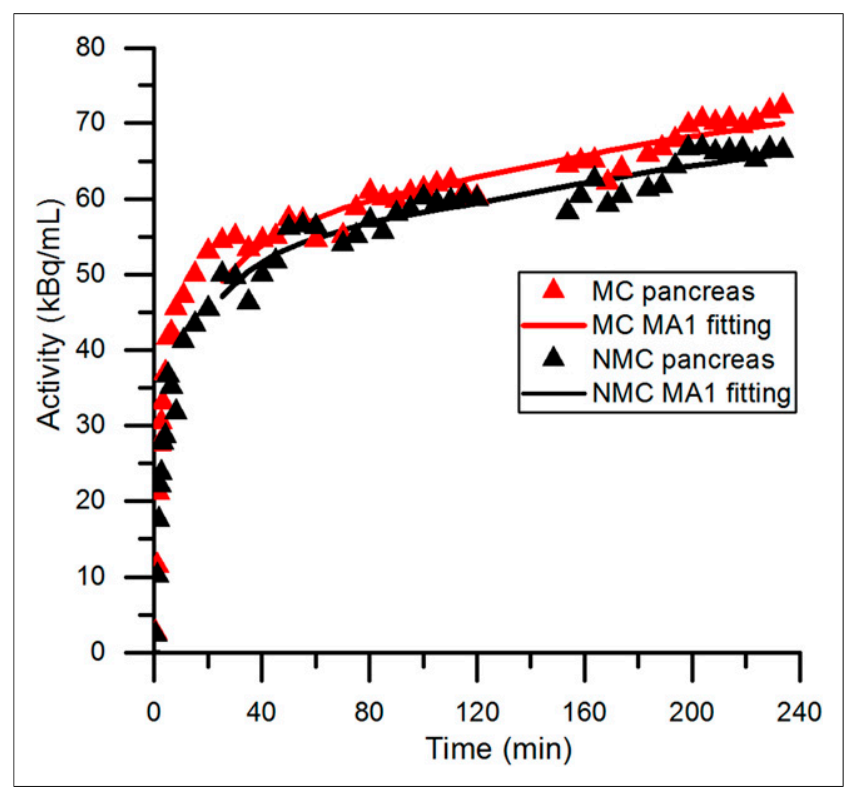

FIGURE 4. Time-activity curves of pancreas for both MC and NMC analyses of subject 8. Data with MC are displayed in red, and data without MC are in black. Symbols show ROI values, and solid lines give multilinear analysis 1 (MA1) fitting results $\left(t^{\star}=20 \mathrm{~min}\right)$. 
TABLE 4

$V_{\mathrm{T}}$ and $K_{\mathrm{i}}$ Values and SD for ${ }^{18} \mathrm{~F}-\mathrm{FP}(+) \mathrm{DTBZ}$ and FMISO Subjects, Respectively, As Well As Percentage Difference Comparing MC with NMC

\begin{tabular}{|c|c|c|c|c|c|c|}
\hline Subject no. & $\begin{array}{l}\text { Motion amplitude } \\
(\mathrm{mm})\end{array}$ & $\mathrm{NMC}, V_{\mathrm{T}}$ & $\mathrm{MC}, \mathrm{V}_{\mathrm{T}}$ & $\mathrm{NMC}, K_{\mathrm{i}}$ & $\mathrm{MC}, K_{\mathrm{i}}$ & Percentage difference \\
\hline 8 & 12.6 & $76.7 \pm 4.0$ & $94.8 \pm 3.7$ & & & $24 \%$ \\
\hline 9 & 10.3 & $159.0 \pm 12.7$ & $187.5 \pm 11.2$ & & & $18 \%$ \\
\hline 10 & 9.7 & $91.5 \pm 6.8$ & $97.4 \pm 1.6$ & & & $6 \%$ \\
\hline 11 & 7.4 & $218.9 \pm 5.1$ & $259.1 \pm 6.9$ & & & $18 \%$ \\
\hline Average & 9.9 & 136.5 & 159.7 & & & $17 \%$ \\
\hline 12 & 5.2 & & & $0.0045 \pm 0.0011$ & $0.0054 \pm 0.0010$ & $20 \%$ \\
\hline
\end{tabular}

only to static PET images. Our results demonstrate that respiratory motion can lead to a significant underestimation of kinetic parameters and INTEX MC can significantly improve the parameter estimation.

In long dynamic PET acquisitions, subjects are more likely to have changes in their breathing pattern and amplitude, introducing time-dependent blurring effects that can further confound the parameter estimation. For example, in the ${ }^{18} \mathrm{~F}-\mathrm{FMISO}$ study (Fig. 5 ), the average motion amplitude of the lung tumor changed from 3.9 to $6.5 \mathrm{~mm}$, which led to a greater increase in the values from the tumor time-activity curve in the second scan (150-180 min), resulting in an overall change in shape of the time-activity curve with MC. These results indicated that INTEX MC could be particularly helpful for long dynamic PET studies when subjects demonstrate variation in their breathing pattern.

Body motion is another factor that can substantially affect quantification of dynamic images. In our study, the ROIs were manually repositioned in both $\mathrm{NMC}$ and $\mathrm{MC}$ analyses for dynamic

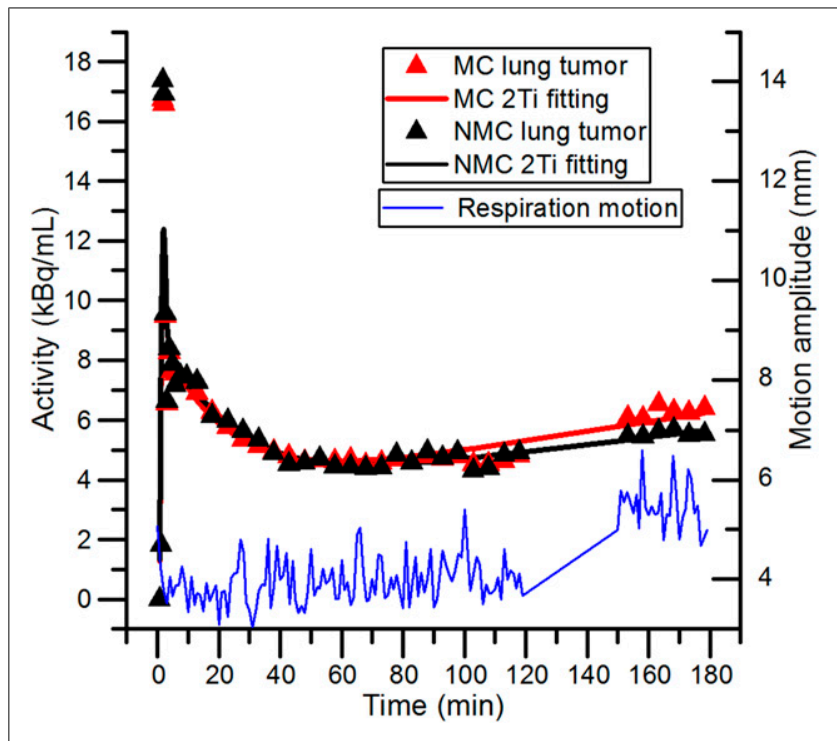

FIGURE 5. Time-activity curves of tumor in lung for both $\mathrm{MC}$ and NMC analyses of subject 12. Data with $M C$ are displayed in red, and data without MC are in black. Triangles indicate activity in tumor for each dynamic frame. Solid lines show 2-tissue irreversible (2Ti) fitting results. Blue solid line shows respiratory motion amplitude averaged over 1-min intervals calculated from Anzai traces and internal-external correlation. frames, in which a visible mismatch was detected to compensate for body motion.

In ${ }^{82} \mathrm{Rb}$ cardiac studies, the responses to regadenoson varied among study subjects. Motion amplitudes increased from the rest to stress studies for subjects $1-3$ by $90 \%$. However, for subjects $4-7$, the motion amplitude increase was only $18 \%$ on average. The individual motion amplitude differences in response to the vasodilators can confound CFR quantification. For subjects 1-3 with significant respiratory motion, the increase of CFR was $34 \%$ on average with MC, and for subjects 4-7 with minimal respiratory motion, the increase was only $7 \%$. The full impact of the changes in a patient's breathing pattern and the benefit of respiratory MC require further investigation, including the evaluation of other pharmacologic stressors currently used in clinical practice.

Compared with PET imaging studies using ${ }^{18} \mathrm{~F}$-labeled radiotracers, cardiac ${ }^{82} \mathrm{Rb}$ PET studies have relatively poorer resolution due to a large positron range of ${ }^{82} \mathrm{Rb}$. MC generally did not seem to substantially change $K_{1}$ and MBF for resting scans. However, for stress scans these changes were substantial, particularly for subjects with motion amplitudes larger than $8 \mathrm{~mm}$. These results indicated

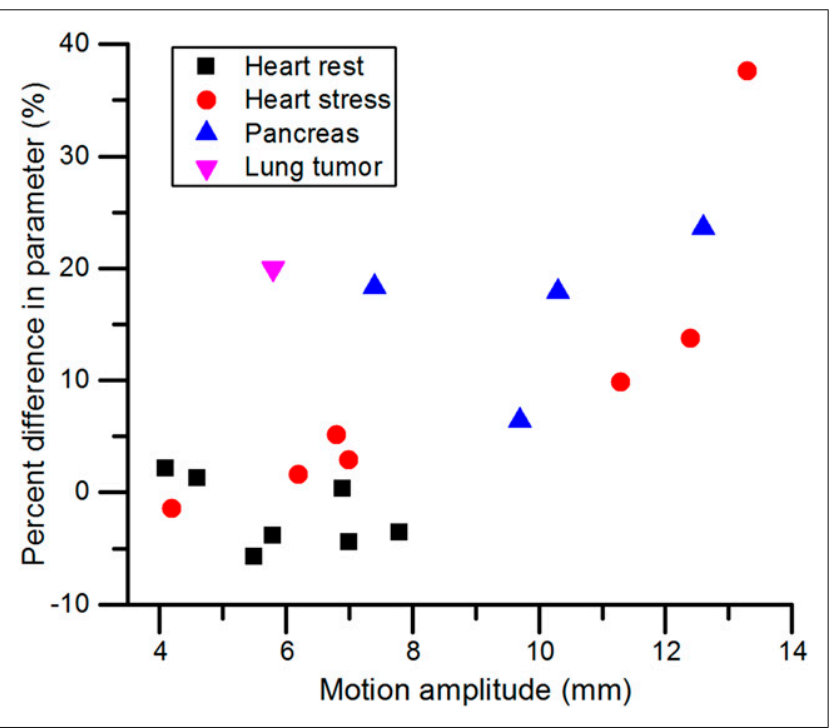

FIGURE 6. Percentage difference in $K_{1}$ for ${ }^{82} \mathrm{Rb}$ (black square for rest studies and red circle for stress studies), $V_{\mathrm{T}}$ for ${ }^{18} \mathrm{~F}-\mathrm{FP}(+) \mathrm{DTBZ}$ (blue triangle), and $K_{\mathrm{i}}$ for ${ }^{18} \mathrm{~F}$-FMISO (pink triangle) for all 12 subjects between $\mathrm{MC}$ and NMC analyses related to motion amplitude of each subject. 
that $\mathrm{MC}$ is essential for subjects with motion amplitudes greater than $8 \mathrm{~mm}$ in ${ }^{82} \mathrm{Rb}$ cardiac PET studies. In the ${ }^{18} \mathrm{~F}-\mathrm{FP}(+) \mathrm{DTBZ}$ and ${ }^{18} \mathrm{~F}$-FMISO studies, MC led to substantially increased parameters $\left(V_{\mathrm{T}}\right.$ and $\left.K_{\mathrm{i}}\right)$ for all subjects, indicating the importance of $\mathrm{MC}$ in the thorax and abdomen for ${ }^{18} \mathrm{~F}$-labeled tracers that demonstrate better resolution.

One limitation of this study is that no true gold standard parameters were available for validation of the benefit of MC. That being said, considering that uncorrected motion introduces blurring, an increase of radiotracer concentration and alterations in the kinetic parameters $\left(K_{1}, V_{\mathrm{T}}, K_{\mathrm{i}}\right)$ shown in this study indicates that our proposed MC should provide more accurate parameter estimation. Specifically, because the kinetic parameters all are reflective of the scale of uptake, reductions in absolute levels in the time-activity curves due to uncorrected motion would reduce these kinetic parameters. This same effect was further investigated and confirmed by simulation studies provided in the supplemental data.

The INTEX/MOLAR approach used in this study is an eventby-event respiratory MC method that rigidly relocates the line of response of each event in a list-mode image reconstruction framework. However, more complex deformation of target organs with larger size will not be fully corrected by a rigid MC method. Specifically, other tissues or organs with different motion amplitudes and patterns may be blurred by rigid MC. We have developed a nonrigid event-by-event MC method for PET with motiondependent point-spread function (21), which more accurately compensates for nonrigid motion. Further investigations are needed to apply this nonrigid INTEX method to dynamic studies.

\section{CONCLUSION}

Respiratory motion can substantially affect parameter estimation in dynamic PET imaging. The INTEX respiratory MC method using continuous external motion signals was shown to substantially change the estimated parameters from dynamic PET of 3 radiotracers with different clinical tasks. More accurate parameter estimation is expected with INTEX MC. Correction for respiratory motion is important for accurate quantification for dynamic PET, particularly for subjects with larger motion amplitudes.

\section{DISCLOSURE}

The costs of publication of this article were defrayed in part by the payment of page charges. Therefore, and solely to indicate this fact, this article is hereby marked "advertisement" in accordance with 18 USC section 1734 . This work was supported by a research contract from Siemens Medical Solutions. This work was also supported by NIH grant 1S10OD010322-01 and by CTSA grant UL1 TR000142 from the National Center for Advancing Translational Science, a component of the National Institutes of Health (NIH). The contents of this study are solely the responsibility of the authors and do not necessarily represent the official view of NIH. No other potential conflict of interest relevant to this article was reported.

\section{ACKNOWLEDGMENTS}

We thank Eric Ackah, MD, PhD, and Herman Feringa, MD, for performing the stress testing in the ${ }^{82} \mathrm{Rb}$ PET imaging.

\section{REFERENCES}

1. El Naqa I, Low DA, Bradley JD, Vicic M, Deasy JO. Deblurring of breathing motion artifacts in thoracic PET images by deconvolution methods. Med Phys. 2006;33:3587-3600.

2. Callahan J, Binns D, Dunn L, Kron T. Motion effects on SUV and lesion volume in 3D and 4D PET scanning. Australas Phys Eng Sci Med. 2011;34:489-495.

3. Liu C, Pierce LA, II, Alessio AM, Kinahan PE. The impact of respiratory motion on tumor quantification and delineation in static PET/CT imaging. Phys Med Biol. 2009;54:7345-7362.

4. Rahmim A, Rousset O, Zaidi H. Strategies for motion tracking and correction in PET. PET Clin. 2007;2:251-266.

5. Nehmeh SA, Erdi Y, Pan T, et al. Quantitation of respiratory motion during 4DPET/CT acquisition. Med Phys. 2004;31:1333-1338.

6. Fulton RR, Meikle SR, Eberl S, Pfeiffer J, Constable CJ. Correction for head movements in positron emission tomography using an optical motion-tracking system. IEEE Trans Nucl Sci. 2002;49:116-123.

7. Lamare F, Carbayo ML, Cresson T, et al. List-mode-based reconstruction for respiratory motion correction in PET using non-rigid body transformations. Phys Med Biol. 2007;52:5187-5204.

8. Li T, Thorndyke B, Schreibmann E, Yang Y, Xing L. Model-based image reconstruction for four-dimensional PET. Med Phys. 2006;33:1288-1298.

9. Chan C, Jin X, Fung EK, et al. Event-by-event respiratory motion correction for PET with 3D internal-1D external motion correlation. Med Phys. 2013;40:112507.

10. Liu C, Alessio AM, Kinahan PE. Respiratory motion correction for quantitative PET/CT using all detected events with internal-external motion correlation. Med Phys. 2011;38:2715-2723.

11. Carson RE, Barker WC, Liow J-S, Johnson C. Design of a motion-compensation OSEM list-mode algorithm for resolution-recovery reconstruction for the HRRT. IEEE Nucl Sci Symp Conf Rec. 2003;5:3281-3285.

12. Jin X, Chan C, Mulnix T, et al. List-mode reconstruction for the Biograph mCT with physics modeling and event-by-event motion correction. Phys Med Biol. 2013;58:5567-5591.

13. Lortie M, Beanlands RS, Yoshinaga K, Klein R, DaSilva JN. Quantification of myocardial blood flow with ${ }^{82} \mathrm{Rb}$ dynamic PET imaging. Eur J Nucl Med Mol Imaging. 2007;34:1765-1774.

14. Normandin MD, Petersen KF, Ding Y-S, et al. In vivo imaging of endogenous pancreatic $\beta$-cell mass in healthy and type 1 diabetic subjects using ${ }^{18} \mathrm{~F}$-fluoropropyldihydrotetrabenazine and PET. J Nucl Med. 2012;53:908-916.

15. Carlin S, Humm JL. PET of hypoxia: current and future perspectives. J Nucl Med. 2012;53:1171-1174.

16. Jakoby BW, Bercier Y, Conti M, Casey M, Bendriem B, Townsend D. Physical and clinical performance of the mCT time-of-flight PET/CT scanner. Phys Med Biol. 2011;56:2375-2389.

17. Martí-Climent J, Prieto E, Domínguez-Prado I, et al. Contribution of time of flight and point spread function modeling to the performance characteristics of the PET/CT Biograph mCT scanner. Rev Esp Med Nucl Imagen Mo (English ed.). 2013;32:13-21.

18. Chan TF, Vese L. Active contours without edges. IEEE Trans Image Process. 2001;10:266-277.

19. Naganawa M, Petersen K, Ding Y-S, et al. Comparison of methods for PET quantification of beta cell mass with $\left[{ }^{18} \mathrm{~F}\right] \mathrm{FP}-(+)-\mathrm{DTBZ}$ [abstract]. $J$ Nucl Med. 2012;53(suppl 1):547.

20. Thorwarth D, Eschmann SM, Paulsen F, Alber M. A kinetic model for dynamic $\left[{ }^{18} \mathrm{~F}\right]$-Fmiso PET data to analyse tumour hypoxia. Phys Med Biol. 2005;50:22092224.

21. Chan C, Jian Y, Germino M, Carson R, Liu C. Non-rigid event-by-event motion correction for PET with motion-dependent PSF. Paper presented at: The 2014 IEEE Nuclear Science Symposium and Medical Imaging Conference; November 8-15, 2014; Seattle, WA. 